\title{
Synthesis and absorption properties of small-molecule acceptor based rich-electronic benzodiselenophene core with hybrid bromination and fluorination end-group
}

\author{
Lai Wang ${ }^{1}$, Jin-Liang Wang ${ }^{1 *}$ \\ ${ }^{1}$ School of Chemistry and Chemical Engineering, Beijing Institute of Technology, Beijing 100081, China.
}

\begin{abstract}
At present, dihalogenated 1,1-dicyanomethylene-3-indanone (IC) have attracted widely attention as terminal unit. Here, our work designed and synthesized a new nonfullerene small molecule acceptor material BDSe-FBr, with simultaneously fluorinated and brominated terminal. Due to that bromine atom has more electrons and larger size, and fluorine atom enhanced intermolecular $\pi-\pi$ packing, BDSe-FBr present strong absorption abilities in solution and film. It shows a broad absorption range of 600-800 nm and 700$900 \mathrm{~nm}$, with the maximum absorption peak in solution and film of $742 \mathrm{~nm}$ and $774 \mathrm{~nm}$, respectively, and the optical band gap of BDSe-FBr is $1.40 \mathrm{eV}$. The results are beneficial for high JSC and less energy loss and these strong absorption abilities are due to that bromine atom has more electrons and larger size, and fluorine atom enhanced intermolecular $\pi-\pi$ packing. The multiple interactions between heteroatom also is beneficial to improve charge transportation and crystallinity. This new A-D-A type small molecules with simultaneously fluorinated and brominated end-group provide an effective strategy to improve photovoltaic performance of acceptor. The introduction of fluorine and bromine atoms simultaneously into the terminal group exhibits great prospects for application in high performance OSCs.
\end{abstract}

\section{Introduction}

Organic solar cells (OSCs) have been attracted more attention, since unique advantages for light weight, flexible, large area fabrication, and solution processability. ${ }^{[1-5]}$ In the past few decades, nonfullerene small molecular acceptor have made significant progresses, benefited from strong absorption and the characteristic of easily chemical modification, especially after Y6 acceptors emerged ${ }^{[6-9]}$ The power conversion efficiencies (PCEs) based on nonfullerene small molecular acceptors have exceeded $18 \%$, which shows expected application potential. ${ }^{[10-13]}$

The acceptor-donor-acceptor (A-D-A) type acceptors have become the most successful molecular structure. This molecular framework is divided into the fused electron-rich core (D) and the electron-withdrawing terminals (A). Benzo[1,2-b:4,5-b']diselenophene (BDSe) as a rich-electron core have been reported in our past work and this strategy for introducing the more electron-rich Se atoms enhances the $\pi$ electron conjugation. BDSe core can also improve the electron mobility and optimize molecular crystal characteristics. ${ }^{[14]}$ Moreover, heteroatom with more electron number enhanced intermolecular interaction, which results in the small bandgap and energy loss..$^{[15]}$

Halogenated 1,1-dicyanomethylene-3-indanone (IC) as an end-group unit plays an important role, in promoting the development of A-D-A type acceptors16. Halogen atoms enable deepening the frontier energy levels of acceptors and widening absorption ability, in addition to promote noncovalent interactions and aggregation abilities, which was caused by high electronegativity and effective electronic delocalization. ${ }^{[17]}$ Thus acceptors with halogenation terminals have exhibited potential ability for fine-tuning the crystalline and optical physical properties. ${ }^{[11,18,19]}$ Electron affinity of different halogens could bring about the complicated influence on the absorption property. In addition, there exist large fluorine and bromine atom large difference. ${ }^{[20,21]}$ Bromine have more rich-electron further to improve absorption abilities and reduce energy loss. While fluorine with most strong electronegativity enhance intramolecular electronic transportation and pacing orientation. ${ }^{[20]}$ However, there are few studies on acceptor material with for electron-rich core and fluorinated and brominated end groups. Based on these, we combined the electron-rich BDSe core with simultaneously fluorinated and brominated terminals and synthesized a new A-D-A type acceptor named BDSe$\mathbf{F B r}$, which achieved strong absorption abilities.

* Corresponding author: jinlwang@bit.edu.cn 


\section{Synthesis}

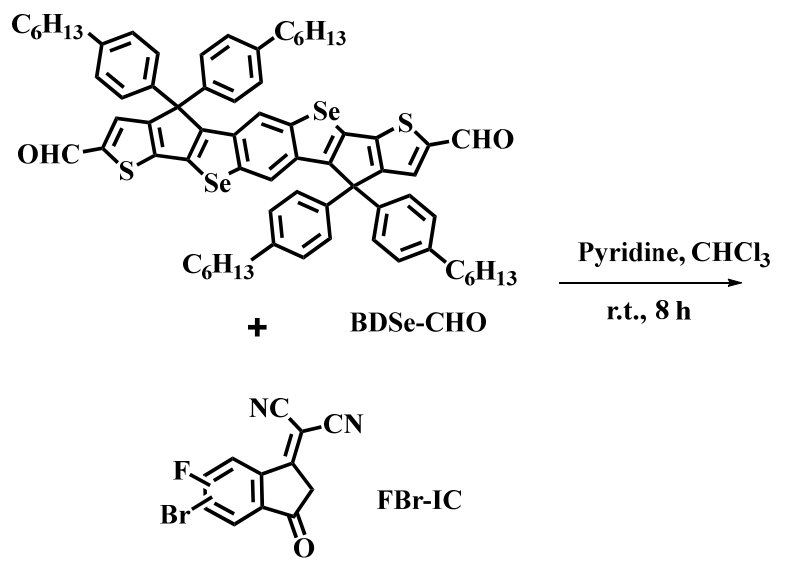

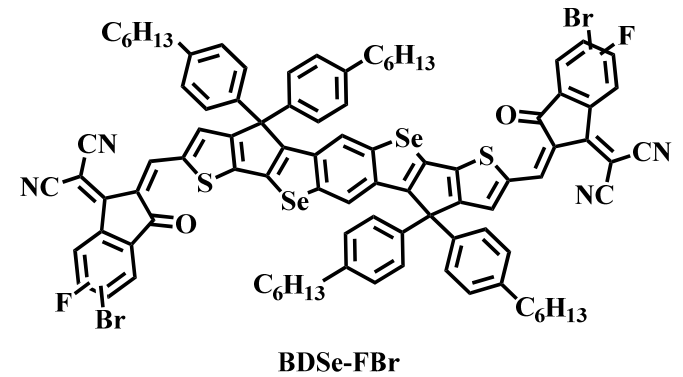

Scheme 1. Synthetic route of BDSe-FBr

Compound BDSe-CHO was synthesized in accordance with past work of our group and FBr-IC was obtained by referring the reported literature. ${ }^{[14,22]}$ As shown in Scheme 1, BDSe-FBr was afforded via the Knoevenagel condensation of BDSe-CHO with FBr-IC in $86 \%$ yield. In detail, FBr-IC (0.26 g, $0.93 \mathrm{mmol})$ and BDSe-CHO $(0.20 \mathrm{~g}, 0.18 \mathrm{mmol})$ were added into a $50 \mathrm{~mL}$ two-neck round-bottom flask, and the mixture was evacuated and backfilled with $\mathrm{N}_{2}$ three times. Then freshly degassed chloroform $(30 \mathrm{~mL})$ and pyridine $(0.4 \mathrm{~mL})$ were added into the reaction mixture. The mixture was stirred at room temperature for $8 \mathrm{~h}$, after that the solution was poured into methanol and the precipitate was filtered off and washed with methanol. The crude product was further to purify by silica gel column chromatography, through eluting with petroleum ether/dichloromethane (1:1) to give a black solid as pure product $(0.16 \mathrm{~g}, 86 \%)$.

The compound BDSe-FBr with three isomers was characterized by ${ }^{1} \mathrm{H}$ NMR in Figure 1. The determined ratio of the three isomers for $\mathbf{B D S e - F B r}$ is ca. 0.02: 0.72: 0.26 , which is based on that hydrogens ratio in end-groups is ca. 1.7: 0.3 from the ${ }^{1} \mathrm{H} \mathrm{NMR}$ spectra. ${ }^{1} \mathrm{H} \mathrm{NMR}\left(\mathrm{CDCl}_{3}\right.$, $400 \mathrm{MHz}, \mathrm{ppm}): \delta 8.85$ (s, 2H, $\left.=\mathrm{CH}), 8.73-8.75 \mathrm{CH}_{3}\right)$. (d, $J=2.8 \mathrm{~Hz}, 0.3 \mathrm{H}, \mathrm{Ph}-\mathrm{H}), 8.43-8.46$ (d, $J=2.8 \mathrm{~Hz}, 1.7 \mathrm{H}$, $\mathrm{Ph}-\mathrm{H}), 8.03$ (s, 2H, Th-H), 7.90-7.91 (d, $J=6.8 \mathrm{~Hz}, 1.7 \mathrm{H}$, $\mathrm{Ph}-\mathrm{H}), 7.68$ (s, 2H, Ph-H), 7.59-7.61 (d, $J=6.8 \mathrm{~Hz}, 0.3 \mathrm{H}$, Ph-H), 7.09-7.16 (m, 8H, Ph-H), 2.54-2.56 (t, $J=7.6 \mathrm{~Hz}$, $\left.8 \mathrm{H}, \mathrm{CH}_{2}\right), 1.55-1.59\left(\mathrm{~m}, 8 \mathrm{H}, \mathrm{CH}_{2}\right), 1.26-1.35(\mathrm{~m}, 24 \mathrm{H}$, $\left.\mathrm{CH}_{2}\right), 0.84-0.87\left(\mathrm{~m}, 12 \mathrm{H}, \mathrm{CH}_{3}\right)$.

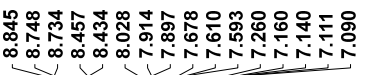

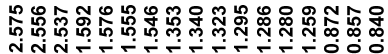
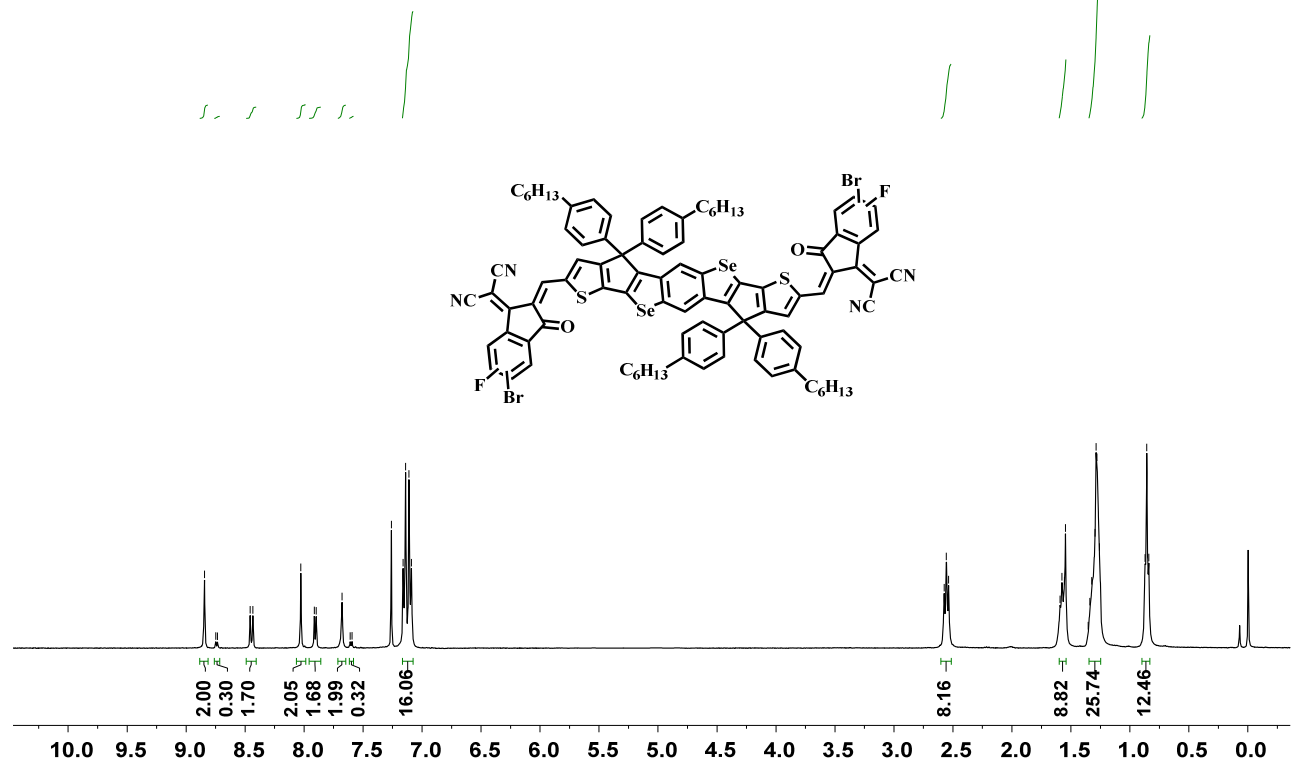

Figure 1. ${ }^{1} \mathrm{H}$ NMR spectra of BDSe-FBr 


\section{Results and discussion.}

BDSe-FBr has good solvent properties in conventional solvents, for instance dichloromethane, chloroform and tetrahydrofuran. The UV-Visible absorption spectrum of BDSe-FBr in solution and film state was displayed in the Figure 2, and the detailed data was listed in Table 1.

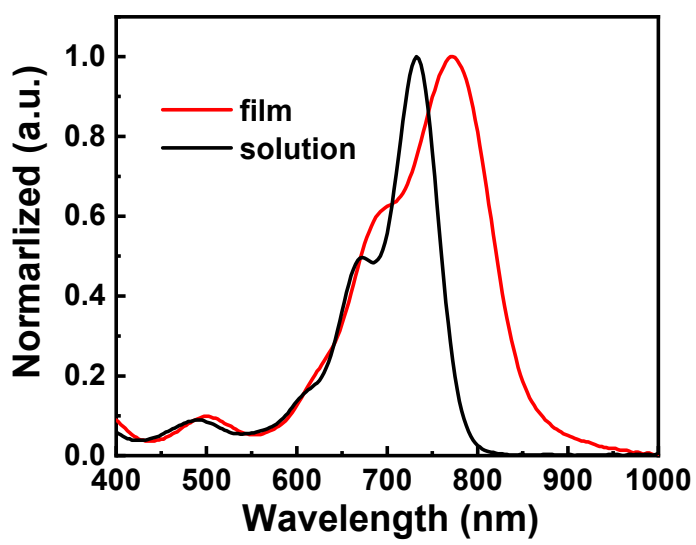

Figure 2. The UV-visible absorption spectrum of BDSe-FBr in solution and film state.

Photophysical properties of acceptor material are usually related with molecular structure, thus UV-Visible absorption spectrum of the acceptor material was carried out in chloroform solution and the thin film in order to explore the relationship. As shown in Figure 2, the BDSeFBr in the chloroform solution displays a broad absorption range of $600-800 \mathrm{~nm}$, and present the maximum absorption at $741 \mathrm{~nm}$.

However, the BDSe-FBr in the film state displays a broader absorption range of 700-900 $\mathrm{nm}$ than solution absorption, and the maximum absorption peak in film state is red-shifted to $774 \mathrm{~nm}$. This obvious red-shift phenomenon could be attributed to the enhanced $\pi-\pi$ stacking effect between acceptor molecules from the solution to the film state, which suggests the introduction of fluorine and bromine atoms improves the intermolecular stacking.

The optical band gap of BDSe-FBr was calculated as $1.40 \mathrm{eV}$ from the edge absorption in film. Small optical band gap is conductive to less energy loss. Moreover, the range of the absorption spectrum of the acceptor show a good complementary when matched with the absorption range of the wide band gap donor PM6, and this is beneficial to improve the photovoltaic performance of the OSC device.

In addition, BDSe-FBr display a most strong absorption ability in chloroform solution, when is compared by acceptors with BDSe core. This result could be attributed to $\mathrm{F}$ atoms have strongest electronegativity than other halogens and hydrogen. Higher electronegativity in end groups will enhanced intramolecular charge transportation and electron delocalization. Thus, fluorinated BDSe-FBr present a most large maximum in solution.

However, the maximum absorption of BDSe-FBr in film is smaller than other acceptors based on BDSe core and halogenated end groups, as in Table 1. It indicates that acceptor with hybrid bromination and fluorination end-group achieves a less $\pi$ - $\pi$ packing and crystallinity. Because fluorine atoms have less number of electrons and atomic orbitals. Small crystallinity is beneficial to reduce charge recombination. This result suggests that BDSeFBr have a strong absorption ability and appropriate crystallization tendency.

\section{Conclusion}

In conclusion, we first designed and synthesized a novel nonfullerene small molecule acceptor material BDSe-FBr, with simultaneously fluorinated and brominated terminals. BDSe-FBr displays a broad absorption range of 600-800 $\mathrm{nm}$, with the maximum absorption peak in solution of $742 \mathrm{~nm}$. When in the thin film, BDSe-FBr present obvious red-shift in the absorption range of 700-900 nm, and maximum absorption shows the same tendency. In addition, the optical band gap of BDSe-FBr is $1.40 \mathrm{eV}$ which was calculated from film edge absorption. These strong absorption abilities are due to that bromine atom has more electrons and larger size, and fluorine atom enhanced intermolecular $\pi-\pi$ packing. And the multiple interactions between heteroatom also is beneficial to improve charge transportation and crystallinity. This new A-D-A type small molecules with simultaneously fluorinated and brominated end-group provide an effective strategy to improve photovoltaic performance of acceptor, and this modification exhibits great future prospects for application in high performance OSCs.

Table 1. The optical properties of acceptors with BDSe core.

\begin{tabular}{cccccc}
\hline Acceptor & $\lambda_{\max }{ }^{\text {sol. }}[\mathrm{nm}]$ & $\lambda_{\max }{ }^{\text {film }}[\mathrm{nm}]$ & $\lambda_{\text {onset }}{ }^{\text {film }}[\mathrm{nm}]$ & $E_{\mathrm{g}}{ }^{\text {opt }}[\mathrm{eV}]$ & Reference \\
\hline BDSe-FBr & 741 & 774 & 886 & 1.40 & this work \\
\hline BDSeIC & 710 & 746 & 816 & 1.51 & {$[14]$} \\
\hline BDSeIC2Br & 726 & 790 & 876 & 1.41 & {$[15]$} \\
\hline BDSe-2(BrCl) & 736 & 793 & 890 & 1.39 & {$[15]$} \\
\hline
\end{tabular}




\section{Acknowledgments}

The authors thank Analysis \& Testing Center (BIT) for NMR experiments.

\section{References}

1. L. Duan, A. Uddin, Adv. Sci., 7, 1903259 (2020).

2. K. Fukuda, K. Yu and T. Someya, Adv. Energy Mater., 10, 2000765 (2020).

3. D. Li, L. Zhu, X. Liu, W. Xiao, J. Yang, R. Ma, L. Ding, F. Liu, C. Duan, M. Fahlman, Q. Bao, Adv. Mater., 32, 2002344 (2020).

4. D. Wang, R. Qin, G. Zhou, X. Li, R. Xia, Y. Li, L. Zhan, H. Zhu, X. Lu, H. L. Yip, H. Chen, C. Z. Li, Adv. Mater., 32, 2001621 (2020).

5. Y. Xie, Y. Cai, L. Zhu, R. Xia, L. Ye, X. Feng, H. L. Yip, F. Liu, G. Lu, S. Tan, Y. Sun, Adv. Funct. Mater., 30, 2002181 (2020).

6. D. Li, X. Zhang, D. Liu, T. Wang, J. Mater. Chem. A, 8, 15607-15619 (2020).

7. S. Li, C.Z. Li, M. Shi, H. Chen, ACS Energy Lett., 5, 1554-1567 (2020).

8. C. Zhan, X. Zhang and J. Yao, RSC Adv., 5, 9300293026 (2015).

9. J. Zhao, C. Yao, M.U. Ali, J. Miao, H. Meng, Mater. Chem. Front., 4, 3487-3504 (2020).

10. Y. Chang, T.K. Lau, P. C. Y. Chow, N. Wu, D. Su, W. Zhang, H. Meng, C. Ma, T. Liu, K. Li, X. Zou, K.S. Wong, X. Lu, H. Yan, C. Zhan, J. Mater. Chem. A, 8, 3676-3685 (2020).

11. Y. Cui, H. Yao, J. Zhang, T. Zhang, Y. Wang, L. Hong, K. Xian, B. Xu, S. Zhang, J. Peng, Z. Wei, F. Gao, J. Hou, Nat. Commun., 10, 2515 (2019).

12. K. Li, Y. Wu, X. Li, H. Fu and C. Zhan, Sci. China. Chem., 63, 490-496 (2020).

13. Q. Liu, Y. Jiang, K. Jin, J. Qin, J. Xu, W. Li, J. Xiong, J. Liu, Z. Xiao, K. Sun, S. Yang, X. Zhang, L. Ding, Sci. Bull., 65, 272-275 (2020).

14. S.S. Wan, X. Xu, J.L. Wang, G.Z. Yuan, Z. Jiang, G.Y. Ge, H.-R. Bai, Z. Li, Q. Peng, J. Mater. Chem. A, 7, 11802-11813 (2019).

15. S.S. Wan, X. Xu, Z. Jiang, J. Yuan, A. Mahmood, G.Z. Yuan, K.K. Liu, W. Ma, Q. Peng, J.L. Wang, J. Mater. Chem. A, 8, 4856-4867 (2020).

16. S. Dey, Small, 15, e1900134 (2019).

17. F. Yang, C. Li, W. Lai, A. Zhang, H. Huang, W. Li, Mater. Chem. Front., 1, 1389-1395 (2017).

18. S. Dai, F. Zhao, Q. Zhang, T. K. Lau, T. Li, K. Liu, Q. Ling, C. Wang, X. Lu, W. You, X. Zhan, J. Am. Chem. Soc., 139, 1336-1343 (2017).

19. S. Li, L. Zhan, Y. Jin, G. Zhou, T. K. Lau, R. Qin, M. Shi, C. Z. Li, H. Zhu, X. Lu, F. Zhang, H. Chen, Adv. Mater., 32, 2001160 (2020).

20. S.M. Swick, J.M. Alzola, V.K. Sangwan, S.H. Amsterdam, W. Zhu, L.O. Jones, N. Powers-Riggs,
A. Facchetti, K.L. Kohlstedt, G.C. Schatz, M.C. Hersam, M.R. Wasielewski, T.J. Marks, Adv. Energy Mater., 10, 2000635 (2020).

21. H. Wang, T. Liu, J. Zhou, D. Mo, L. Han, H. Lai, H. Chen, N. Zheng, Y. Zhu, Z. Xie, F. He, Adv. Sci., 7, 1903784 (2020).

22. F. Peng, K. An, W. Zhong, Z. Li, L. Ying, N. Li, Z. Huang, C. Zhu, B. Fan, F. Huang, Y. Cao, ACS Energy Lett., 5, 3702-3707 (2020). 